\title{
China y la provisión de bienes públicos globales. Falacia sobre el ascenso hegemónico
}

DOI: $10.32870 /$ mycp.v1i1.386

Eduardo Tzili Apango

\section{Resumen}

1 1 resurgimiento de China como potencia global causa tanto escepticismo como tranquilidad en diversas partes del planeta; desde la viabilidad de disputar la hegemonía mundial a Estados Unidos por medio de la fuerza, hasta precisamente la posibilidad de fungir como un contrapeso importante ante Occidente. En este sentido, y con base en la teoría de la estabilidad hegemónica (TEH), uno de los indicadores para identificar una hegemonía es la capacidad de proveer los llamados bienes públicos globales (BPG). La problemática que da vida al presente estudio parte, eminentemente, de identificar si el abastecimiento de dichos BPG es un rasgo hegemónico. Para lo anterior tomaré como caso de estudio el papel que la República Popular China desempeñó en la crisis asiática de 1997-1998, realizando también un análisis comparativo sobre el rol que ha desempeñado en la crisis financiera global de 2008-2010. La investigación intenta responder a la pregunta: ¿posee China el rasgo hegemónico de la provisión de los BPG? Al final se presentan una serie de consideraciones al respecto, donde se pretende comprobar o falsear la siguiente hipótesis: en la crisis de 1997-1999 China no presentó rasgos hegemónicos con base en la provisión de algún BPG, pero en la crisis de 2008-2010 sí los presenta.

Palabras clave: hegemonía, economía de mercado, estabilidad, bien público internacional, crisis económico-financiera. 


\section{Abstract}

China's resurgence as a global power causes as much skepticism as tranquility in several parts of the world, from the feasibility to challenge U. S. global hegemony by force, to precisely serving as an important counterweight to the West. Based on Hegemonic Stability Theory (HST), one of the indicators to identify hegemony is its ability to provide the so-called Global Public Goods (GPG). The issue that brings to life the present research is, essentially, to identify if the GPG's supplying is hegemony characteristic. To do this, I take as a case of study the role that the People's Republic of China played during 1997-1999 and 2008-2010 financial crisis, making a comparative analysis. The research tries to answer the following question: Does China has the hegemonic trait of supplying GPG? In the end it is showed some final considerations trying to check or distort the next hypothesis: in the 1997-1999 crisis, China did not have hegemonic features based on supplying a GPG, but in the 2008-2010 crisis, China do have.

Keywords: hegemony, market economy, stability, international public good, economic-financial crisis.

\section{Introducción}

El presente artículo de investigación tiene por objetivo indagar en la dinámica de los bienes públicos globales (BPG), cuyo emergente estudio en nuestro país coloca sobre la mesa una posible estructura mundial de la que no nos hemos percatado, y es aquella gobernanza global a través del suministro de dichos bienes en un mundo aparentemente anárquico.

En esta línea de pensamiento, argumento que la naturaleza del poder global cambia lentamente de una realidad en donde no existían organizaciones internacionales, interdependencia y multilateralismo (al menos no de manera tan profunda como en la actualidad), hacia un contexto contemporáneo cuyas características hacen casi imposible, mas no improbable, que se efectúe lo que Gilpin reconoce como "guerras hegemónicas". ${ }^{1}$

Es decir, en un mundo intrínsecamente conectado en múltiples niveles, donde los mecanismos de organización internacional se consolidan poco a poco y cada vez más, la hegemonía mundial ya no se efectuará a través del

1. Gilpin, 1988b. Véase también: Gilpin, 1988a. 
poder duro, sino del poder inteligente, ${ }^{2}$ cuyo componente del poder suave consiste (entre otras cosas) en la capacidad de proveer bienes públicos, como seguridad o conocimiento, pero a escala mundial.

Así, entre más capacidad de provisión de los BPG, más poder se tiene al interior del sistema. Y en la actualidad el único Estado que posee una enorme capacidad de proveer múltiples BPG es Estados Unidos, que aunado a su cuantioso poder duro, lo hacen la indiscutible hegemonía global. Sin embargo, estudiosos afirman que un cambio estructural en el balance de poder global se está llevando a cabo (sobre todo en círculos intelectuales y de poder estadounidenses), ${ }^{3}$ en donde China eventualmente igualará, si no es que excederá, las capacidades de poder de Estados Unidos, "desafiando" su hegemonía; ${ }^{4}$ produciendose así la "teoría de la amenaza china”.

El estudio trata de observar si en efecto China adquiere habilidades hegemónicas, y específicamente aquella que le capacita para proveer bienes públicos globales. Para lo anterior, el escrito se divide en cuatro partes; en la primera se exponen de manera concreta qué son los bienes públicos globales; en la segunda se estudia la participación de China en la crisis financiera asiática de 1997-1999 donde se encuentra que, más que haber contribuido a la estabilidad financiera regional (lo que habla de una habilidad hegemónica de abastecer un bien público global complementario), fue una de las causas; en la tercera parte se estudia la participación de China en la recesión mundial de 2008-2010, donde se visualiza un país más fuerte y posiblemente apto para la provisión de BPG. En la última parte se exponen una serie de consideraciones finales al respecto, así como la identificación de futuras líneas de investigación sobre el tópico.

2. Para una revisión del concepto de "poder inteligente", véanse: Nye, 2011: 207-217; Nossel, 2004.

3. Véase por ejemplo: Gertz, Bill, China Threat. How the People's Republic of China targets America, Regnery Publishing, United States, 2002; Roy, Denny, "The 'China Threat' issue. Major Arguments”, Asian Survey, 36(8), University of California Press, United States, 1996, pp. 758771; Gries Hays, Peter, "A China threat? Power and passion in Chinese 'face nationalism", World Affairs, 162(2), United States, 1999, pp. 63-75; Leeb, Stephen, "The Real China Threat", Times, 2011, en http://ideas.time.com/2011/12/04/the-real-china-threat/, entre otros.

4. Wilkins, Thomas, "The New 'Pacific Century' and the rise of China: an International Relations Perspective", Australian Journal of International Affairs, Routledge Taylor \& Francis Group, Vol. 64, No. 4, 2010, pp. 381-405. 


\section{Bienes públicos globales: delimitación conceptual}

Actualmente hay todo un debate sobre la definición de los BPG. En lo que sí concluyen varios autores es que los BPG, al igual que los bienes públicos domésticos o locales, también contienen los principios de no exclusión y no rivalidad; ${ }^{5}$ su consumo por parte del actor $A$ no implica que no consuma $B$ o $C$, y que su consumo por parte de $A$ no agotará el bien para $B$ o $C$. Lo anterior no se limita a la esfera mundial, ya que puede haber ciertos bienes públicos de carácter regional, cuyo consumo no excluye o rivaliza con otras áreas del planeta, ya que obedece a características propias de la región. ${ }^{6}$

Es difícil la identificación de un BPG puro, ${ }^{7}$ y la llamada "gobernanza global" a través de la red transnacional no está lo suficientemente estructurada como para proveer de un BPG de manera completa e imparcial. Es por ello que definiré a un BPG como aquel bien que puede ser proveído a nivel mundial de manera transnacional y relativa, mas no absoluta.

Ahora bien, se podría considerar un error ligar el abastecimiento de los BPG a la llamada "gobernanza hegemónica", tal como lo hacen Kindleberger y Gilpin. ${ }^{8}$ Es decir, ¿cómo hablar de un BPG si no hay un gobierno mundial que lo suministre? Pero esto obedece a que la teoría de los BPG aún no estaba muy bien desarrollada cuando se proponía la TEH en las relaciones internacionales (de hecho, el debate teórico de los BPG empezó hasta la segunda mitad de la década de 1990); además, referirse a un gobierno mundial no es viable ya que eso aún no ha sucedido.

Oliver et al. ${ }^{9}$ identifican cinco tipos (o sectores) de BPG: ambiente, salud, conocimiento, seguridad y gobernanza, mismos que se pueden alcanzar a partir de actividades básicas y aquéllas complementarias. Las primeras se refieren al abastecimiento (o producción) del BPG en sí, y las segundas a la asistencia que es otorgada para la provisión de dicho bien. Por ejemplo, en el caso de la gobernanza internacional la actividad básica sería aquella que realizan las instituciones multilaterales (la Organización de Naciones Unidas, la más representativa) para la estabilidad mundial, a través de la actividad

5. Véanse: García Arias, 2004: 187-212; Acjkgöz Ersoy, 2011; Oliver, Willem te Velde, y Hewitt, 2002: 31-46; Kaul, Grunberg, y Stern, 1999: 2.

6. Sandler, 1998: 222.

7. García Arias, 2004: 191 y 192.

8. Véanse: Kindleberger, 1986: 1-13; y Gilpin, 1988b.

9. Oliver, Willem te Velde, y Hewitt, 2002: 40 y 41. 
complementaria como la fortaleza de la sociedad civil de los países miembros, o como el fomento de la democracia.

Es importante tomar en cuenta el problema de la financiación de los bienes, lo cual puede desembocar en un problema clásico de las relaciones internacionales: el free-rider, ${ }^{10} \mathrm{y}$ con esto quiero decir que muchas veces es mejor no cooperar en la provisión de un BPG, dejando que el resto de los actores sostengan los costos. Y es que siempre hay países que aportan más que otros, pero no porque no quieran, sino porque a veces sus capacidades no se los permiten. En este tenor, Sandler expone lo que llamó "la paradoja de los BPG":

En la ausencia de un mecanismo de exclusión, una preocupación real existe en cuanto a qué naciones confrontarán a los más grandes contaminantes globales, la amenaza de Estados fallidos, la proliferación de armas nucleares o la inestabilidad de los mercados financieros. Todos estos problemas poseen aspectos de bienes públicos que pueden motivar a muchas naciones en confiar o free-ride en las acciones de otros países. ${ }^{11}$

Es en ese sentido donde entra la necesidad de la hegemonía: un BPG debe ser abastecido; no es opcional porque los costos de no mantener ciertos bienes públicos pueden ser demasiado altos o, incluso, irreversibles. Un ejemplo de ello bien podría ser el cambio climático, el cual es un "mal público global". ${ }^{2}$ Aunque no es el objetivo del presente estudio, es importante recalcar la diferencia entre aquéllos BPG necesarios y los no necesarios, ya que como bien señala Sandler, ${ }^{13}$ hay algunos bienes que pueden no ser abastecidos, aunque para mitigar el impacto de sus consecuencias negativas se proveen de otros no directamente relacionados. Esto también tiene que ver con las capacidades de los Estados y con el costo-beneficio que implica cada bien público.

De igual manera considero importante destacar que en la definición de los BPG no me limito a la esfera puramente geográfica (global, transnacional, local), ya que reconozco su naturaleza multidimensional, que incluye aspectos temporales (los BPG de hoy pueden no ser los de mañana, o viceversa. Ejemplo: la energía nuclear que hoy no es un BPG, probablemente lo será en un futuro) y sociológicos (los BPG pueden ser producidos pensados para determinados

10. Kaul, Grunberg, y Stern, 1999: 6-8.

11. Sandler, 1998: 222.

12. García Arias, 2004: 191.

13. Sandler, 1998: loc. cit. 
objetivos sociales, pero no siempre los cumplen. Ejemplo: los programa de Naciones Unidas para el África subsahariana que se supone se enfoca en erradicar la pobreza pero no a superarla), coincidiendo con Kaul et al. ${ }^{14}$

En el ámbito donde existan Estados incapaces de contribuir a la provisión de BPG, y que esto sea base para la inestabilidad internacional, es donde interviene la hegemonía. Con base en la teoría de la estabilidad hegemónica podría ejemplificar la aseveración anterior con el caso de la Pax Britannica y la "gobernanza hegemónica" que ejerció, proveyendo de estabilidad internacional a través de la regulación financiera con base en el patrón oro, así como la persecución de un mercado autorregulado, aunque también se observa que una hegemonía es capaz de proveer más de un BPG, como es el caso del sistema internacional de balance de poder que imperó durante el siglo XIX para la estabilidad de la política europea, así como el fomento de un Estado liberal. ${ }^{15}$

Otro ejemplo es el de la Pax Americana, caso que después de la incapacidad de Reino Unido para mantener estable al sistema internacional (dando fin a la "paz de los 100 años"), este último colapsó y la transición hegemónica se dio por medio de dos conflagraciones mundiales, así como por una profunda crisis económica (1929). Se dio la necesidad de una nueva hegemonía que fuera capaz de seguir proveyendo determinados BPG para la estabilidad del sistema. Estados Unidos fue aquel actor capaz de proveer un ambiente seguro de cooperación política y económica para la creación de regímenes internacionales que ayudaron a reconstruir la Europa devastada y la economía internacional en general. ${ }^{16}$ Ante el fortalecimiento de una posible competidora por la hegemonía, Washington enfocó esfuerzos para incrementar sus capacidades militares, cuyos despliegues en la Guerra de Corea, de Vietnam y del Golfo Pérsico, aunado ello a las detonaciones de dos bombas nucleares en el Noreste asiático y la posterior sofisticación de sus armas de destrucción masiva, la convirtieron en la indiscutible hegemonía militar, superando por mucho al resto de los actores del sistema mundial.

No obstante, Keohane también argumenta que la TEH se concentra sólo en la oferta de regímenes internacionales; entre más concentrado esté el poder en el sistema internacional, mayor es la capacidad de oferta, aseverando que los gobiernos pueden demandar un régimen internacional debido a una

14. Kaul, Grunberg, y Stern, 1999: 11 y 12 .

15. Polanyi, 2003: 49.

16. Keohane, 2005: 207; y Gilpin, 1990: 86 y 87. 
ineficiencia institucional, que a nivel mundial es resultado de inhibir la coordinación mutuamente ventajosa. La cooperación es posible en la ausencia de una hegemonía porque existe una serie de condiciones específicas que hacen emerger determinado régimen internacional. ${ }^{17}$

Ahora bien, habría que diferenciar entre "régimen" e "institución", ya que pueden ser fácilmente confundibles a la hora de investigar tópicos relacionados con la hegemonía, la cooperación y las relaciones internacionales. Régimen se define como aquella acción que facilita la cooperación, el orden y la estabilidad, además de que ayuda a la institucionalización de porciones de la realidad internacional. ${ }^{18}$ Relacionado con el costo-beneficio, los regímenes son creados por los Estados en función del interés propio y de los beneficios recíprocos que disfrutan al obedecer las reglas que ellos mismos se impusieron. ${ }^{19}$

Institución se refiere a un modelo general o categorización de actividad o arreglo particular construido por el hombre, organizado formal o informalmente, además de que implica persistentes y vinculados conjuntos de reglas que prescriben papeles de comportamiento, restringen la actividad y configuran las expectativas. ${ }^{20}$

En pocas palabras, las instituciones son el conjunto de reglas que articulan y organizan las interacciones económicas, políticas y/o sociales entre Estados, y se construyen históricamente. Los regímenes son acciones que facilitan el cumplimiento de dichas reglas. Una hegemonía puede fomentar un régimen internacional más fácilmente que la promoción de una institución internacional. La hegemonía construye regímenes con base en las instituciones existentes.

A manera de síntesis de este primer apartado, un BPG es aquel que suministra estabilidad al sistema global en sus diversas manifestaciones. Lo ideal es que el mismo fuera producto de la cooperación, pero ésta es asimétrica y no todos los Estados pueden cooperar de la misma manera. Además, existen diversos sectores de los BPG que afectan a múltiples aspectos de la realidad internacional. El que uno falte de ser proveído puede no afectar a los otros, pero sí al sistema. Del mismo modo, se vio que un BPG contiene los principios de no exclusión y no rivalidad. En el financiamiento (o la provisión en sí) de

17. Aunque para este efecto, el mismo autor reconoce que es más fácil mantener un régimen que crearlo, al señalar que en esto último la hegemonía es importante e incluso crucial. Véase: Keohane, 2005: 155-210.

18. Haggard, y Simmons, 1987: 495 y 496.

19. Hurrell, 1992: 651.

20. Keohane, 2005: 242. 
un BPG nos podemos encontrar con el problema del free-rider, por un lado, o la situación de la gobernanza hegemónica, por otro; ambos representan una faceta del suministro de un BPG, como se ve en la figura 1. Por último, un BPG debe ser suministrado a través de un régimen internacional, que bien puede estar o no representado a través de una institución.

\section{Figura 1}

Esquema del problema del abastecimiento de BPG

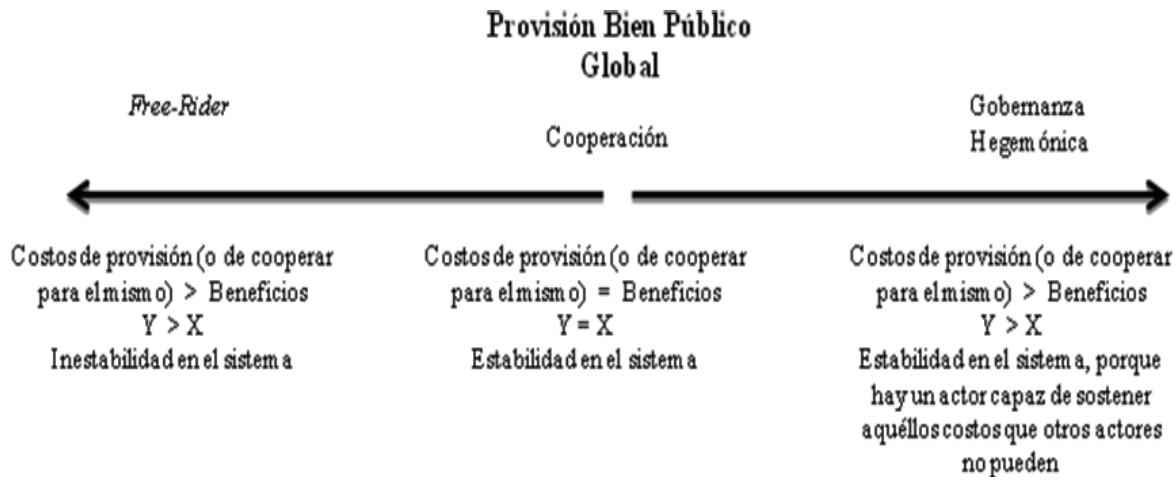

Fuente: elaboración propia con base en Sandler, ${ }^{21}$ García-Arias, ${ }^{22}$ Kindleberger ${ }^{23}$ y Gilpin. ${ }^{24}$

Una vez vista la naturaleza de los BPG, procederé a estudiar el caso de la crisis asiática de 1997, cuando se presentó una inestabilidad en el sistema financiero a causa de la decisión de dejar las monedas al manejo del mercado, lo cual a su vez ocasionó recesiones regionales, inflación y decrecimiento. Adoptando los términos ya expuestos sobre la teoría de los BPG, la región presentó desequilibrios a causa de la falta de aprovisionamiento de BPG de la gobernanza (actividad básica) y la estabilidad financiera (actividad complementaria). Empero, en ese entonces China también fue impactada por dicha crisis, sin capacidades de proveer algún bien público significativo. Es por ello que el bien público tuvo que ser proveído por otros actores, ya que de lo contrario hubiera contagiado a todo el sistema financiero. No sucedió

21. Sandler, 1998.

22. García Arias, 2004.

23. Kindleberger, 1986.

24. Gilpin, 1988b. 
lo mismo en la crisis de 2008, cuando se muestra una China con mayores y mejores capacidades para adoptar un papel activo en la gobernanza regional.

\section{La crisis asiática de 1997 y el papel de China}

Es de mi parecer que las afirmaciones acerca de la mitigación de los efectos de la crisis de 1997 gracias al sostenimiento del valor del yuan y la fortaleza económica de China, merecen revisión..$^{25}$ De acuerdo con Pablo Bustelo, la moneda se apreció respecto al grupo de Asia- $-5,{ }^{26}$ las exportaciones presentaron decrecimientos, disminuyó el superávit comercial y la inversión extranjera directa (IED), aumentó el desempleo, se presentó caída en los precios, hubo menor crecimiento en la reserva de divisas y se efectuó una política monetaria (o cambiaria, más específicamente) más restrictiva. ${ }^{27}$

Asimismo, instituciones internacionales como el Fondo Monetario Internacional (FMI) o el Banco Asiático de Desarrollo (BAD) no pudieron intervenir de manera eficaz para prevenir el colapso financiero que se avecinaba. En segundo término, no hicieron mucho para aminorar las consecuencias de la crisis en la región. ${ }^{28}$

En la dinámica de las transacciones financieras y comerciales, identifico una institución formal e informal a la vez, que en el caso del Este asiático se materializaba en forma de la Asociación de Naciones del Sureste Asiático (ASEAN, por sus siglas en inglés) y en los volúmenes de comercio que presentaban una integración en vías de profundizarse. ${ }^{29}$

Al momento de dispararse la crisis, que ocurrió en razón de la devaluación del bath tailandés, lo que provocó una fuerte inestabilidad cambiaria y bursátil en otros países miembros del ASEAN, el FMI acudió al rescate de Tailandia con 17,200 millones de dólares, y con 23,000 millones de dólares para Indonesia. ${ }^{30}$ Obviamente el paquete de ayuda no estuvo exento de condiciones: incrementos en las tasas de interés, en impuestos, y reducción de gasto guberna-

25. Véase por ejemplo: "A Stable Yuan Contributes to the Global Economic Recovery", China .org. cn, 2010; Kwan, 1998; Jianxin, 2008, entre otras opiniones al respecto.

26. Corea del Sur, Filipinas, Indonesia, Malasia y Tailandia.

27. Véase: Bustelo, 2000: 7-15; y Bustelo, 2001: 66-77.

28. Véase: Sitiglitz, 2003: 89-132; y Bustelo, 1999: 13-18.

29. Para 1995, lo seis principales lugares de IED eran China, Tailandia, Malasia, Indonesia, Taiwán y Singapur, identificándose también el "milagro asiático" por parte del FMI. Véase: López Villafañe, 1999: 99-122.

30. Bustelo, 1999: 12. 
mental, aunado a la exigencia de "reformas estructurales"; medidas de corte neoliberal que debido a la naturaleza de las economías asiáticas, agravaron el problema, más que solucionarlo. ${ }^{31}$ Se considera que el "modelo asiático" no se apegaba a los modelos teóricos de Krugman y Obstfield (primera y segunda generación); ${ }^{32}$ es decir, que no era ortodoxia económica capitalista. De hecho hay autores que piensan que su abandono fue el directamente responsable de las debilidades presentadas. ${ }^{33}$

El régimen creado para la estabilización de la balanza de pagos no pudo asistir a la resolución de la crisis financiera asiática de 1997. La institución financiero-comercial mundial, no obstante, se mantuvo relativamente estable porque a diferencia de la crisis de 1929, cuando el alcance fue global, el esfuerzo internacional logró mantener focalizados los efectos a nivel regional, sin que por ello tuviera ciertas repercusiones internacionales. ${ }^{34}$

China, en este sentido, más que ir al rescate de la región, podría decirse que fue una causa indirecta. Como lo demuestra $\mathrm{He},{ }^{35}$ la fuga de capitales, disfrazada a manera de inversiones, sumada a la deuda de las empresas estatales y a la falta de un mecanismo externo para regular efectivamente la administración de valores, fueron pequeños golpes para el estallido de la crisis.

Beijing trató de proteger al país, lo que logró relativamente, pero sus medidas macroeconómicas no tuvieron grandes repercusiones regionales, ni qué decir externas; se notó un rol potencial del control de capitales por parte del Gobierno chino que contribuyó a la estabilidad, pero ello previno que instituciones financieras chinas prestaran capital de manera excesiva al exterior, ${ }^{36}$ lo que se suma a la vinculación del dólar por parte del yuan a partir de 1997, estrategia que sólo siguió Malasia, pero que igual causó la inestabilidad monetaria regional ya conocida. Luego entonces, China no tuvo la capacidad de proveer el bien público complementario "estabilidad financiera", lo cual no presentó un rasgo hegemónico.

31. Sitiglitz, 2003: 96 у 97.

32. Bustelo, 2000.

33. Singh, 1998: 14.

34. Bustelo, 1999: 13.

35. Qinglian, 1997.

36. Fernald y Babson, 1999. 
China y la provisión de bienes públicos globales. Falacia sobre el ascenso hegemónico

Cuadro 1

Valor del yuan y de las monedas del Asia-5

\begin{tabular}{lrrrrr}
\hline & 1995 & 1996 & 1997 & 1998 & \multicolumn{1}{c}{1999} \\
\hline Yuan renmimbi & 12.36 & 11.99 & 8.27 & 8.27 & 8.27 \\
Won coreano & $1,145.01$ & $1,206.78$ & 1,355 & $1,221.15$ & $1,141.80$ \\
Peso filipino & 38.91 & 37.92 & 34.95 & 43.8 & S/D \\
Rupia indonesa & $3,417.81$ & $3,376.83$ & 5,560 & 14,850 & S/D \\
Ringgit malayo & 3.77 & 3.63 & 3.68 & 3.8 & 3.8 \\
Bath tailandés & 37.36 & 36.75 & 42.88 & 35.9 & 38.71 \\
\hline
\end{tabular}

Los valores expresados son el promedio de lo registrado mensualmente en diciembre del año respectivo. Los valores son respecto al dólar estadounidense.

Fuente: Fondo Monetario Internacional (http://www.imf.org/external/np/fin/data/param_ rms_mth.aspx).

$\mathrm{Al}$ no tener capacidades hegemónicas, no hubo oferta de régimen internacional en materia financiera. Sin embargo, la situación tenía que ser controlada. Ante ello se creó el grupo de los $20^{37}$ y se propuso la Iniciativa Chiang-Mai con el fin de atemperar los efectos de la crisis, lo cual deja entrever que hubo una demanda de régimen internacional, cumpliéndose lo señalado por Keohane en líneas anteriores.

En este sentido, el modelo del "vuelo de los gansos" bajo la égida japonesa estaba fomentando lo que Fawcett y Hurrell ${ }^{38}$ identifican como "regionalización", así como "conciencia e identidad regional". Al intentar integrarse a la economía global adoptando esquemas de tipo más neoliberal (que se podría decir, era la institución "de moda"), provocó una crisis que repercutió en la configuración regional, al grado de que Japón perdió la relativa hegemonía regional que poseía, dando paso a China, país que no "aprovechó" el vacío hegemónico regional. Un ejemplo de ello es la propuesta por parte de Tokio para la creación de un Fondo Monetario Asiático, rechazada inmediatamente por Estados Unidos y el FMI. China no mostró entusiasmo al respecto. En su

37. Creado en 1999 en el ocaso de la crisis asiática, se conforma por: Alemania, Arabia Saudí, Argentina, Australia, Brasil, Canadá, China, Corea del Sur, Estados Unidos, Francia, India, Indonesia, Italia, Japón, México, Rusia, Reino Unido, Sudáfrica, Turquía y la Unión Europea, a través del Foro de Estabilidad Financiera. Véase: "History", Financial Stability Board, en http://www.financialstabilityboard.org/about/history.htm

38. Fawcett y Hurrell, 1995: 38-42. 
lugar se propuso, como ya se dijo en párrafo anterior, la Iniciativa Chiang $\mathrm{Mai}^{39}$ por parte del ASEAN. ${ }^{40}$

Así, en la crisis financiera asiática de 1997 Beijing aún no contaba con las capacidades de proveer el BPG de gobernanza en el ámbito económico. Gracias a sus medidas contracíclicas, China mantuvo cierto grado de estabilidad financiera pero sólo al interior, no teniendo un efecto de spillover (o derrame) por la región.

Por lo mismo, tampoco tuvo la facultad de fomentar un régimen internacional. En su lugar, el G-20 logró convocar a un diálogo más abierto para incorporar a las economías emergentes, estructuró acuerdos para reducir la vulnerabilidad financiera, para instrumentar políticas macroeconómicas más consistentes, mejorar la regulación y supervisión financiera, así como suscribir los estándares y códigos internacionalmente aceptados; ${ }^{41}$ aunque su creación no fue la única opción posible, ${ }^{42}$ al parecer denotó un reflejo más puntual de la configuración de poder a nivel internacional.

La necesidad de reformar las organizaciones internacionales (énfasis en el sistema monetario internacional, o SMI) empujó a los Estados a cooperar, repartiendo los costos de proveer los BPG de gobernanza y estabilidad financiera. Cabría preguntarse si la acción del G-20 resolvió la crisis o sólo la retrasó hasta la experimentada en 2008, cuando se presentaron dificultades estructurales primero en Estados Unidos y luego en Europa, actores que posiblemente ya no soportaron más los costos de mantener un sistema económico-financiero internacional determinado. Y en esta línea, argumento que las capacidades chinas se han incrementado exponencialmente, por lo que la siguiente fase del presente análisis es verificar si ya consiguió potencial para provisión de BPG a través de la creación de regímenes internacionales.

39. Teniendo como antecedentes al Acuerdo de Canjes Asiático (ASA, 1977), el Fondo Monetario Asiático (1997), y la Iniciativa Miyazawa (1998), la Iniciativa Chiang Mai (2000) es un sistema para prevenir una crisis financiera regional por medio del intercambio de moneda.

40. Eichengreen, 2004: 26.

41. Abeles y Kiper, 2010: 5 .

42. Recordar que primero fue G-22 (o Grupo de Willard), luego G-33, además de que se podía extender el Banco de Pagos Internacionales para incluir a las naciones emergentes. Véase: Kim, 2011: 15 y 16. 


\section{La crisis global financiera de 2008 y el papel de China}

La crisis de 2008 inició en Estados Unidos a partir de una burbuja inmobiliaria que terminaría afectando todo el sistema financiero; se volvió global a causa del efecto "contagio" que suponen los títulos con respaldo hipotecario mediante los cuales se "empaquetaron" hipotecas de diversa calidad que se mercadearon en Estados Unidos y Europa principal aunque no únicamente, descubriendo que a partir de 2007 se suscitaron varios problemas en un sistema financiero altamente globalizado y desregulado. Las empresas que tenían en sus balances activos vinculados directa e indirectamente con activos tóxicos, empezaron a sufrir caídas a partir de 2007, mismos que estaban localizados principalmente en los mercados europeos. ${ }^{43}$

En el caso específico de China, se observa que debido a los controles de flujos de capital por parte del gobierno comunista, resultó menos afectada por las variaciones de los tipos de cambio en las monedas, presenciando un repunte significativo del yuan renminbi desde el primer trimestre de 2007, hasta la caída de Lehman Brothers, y de ahí ha mantenido una evolución estable, paralela al dólar. ${ }^{44}$

De igual manera, se ha presentado un impacto menor en materia comercial: si bien la caída del comercio con Estados Unidos fue pronunciada, en 2009 China registró un valor tan sólo de $-9.76 \%$ respecto a 2008 en materia de exportaciones. Comparado por ejemplo con Canadá, que en un marco de Tratado de Libre Comercio redujo sus exportaciones en razón de $-26.73 \%$. Si bien son perceptibles recuperaciones no mayores a $10 \%$ para 2010 para ambos actores, también se puede ver una diversificación más alta sobre el destino de las exportaciones chinas, lo que atemperó los efectos de la crisis en el comercio del país asiático.

En comparación con la crisis asiática de 1997, China fue impactada pero en otras magnitudes; un ejemplo es el caso de la moneda, que mientras entre 1997-1999 el yuan chino registra un saldo negativo de $-0.0002 \%$, entre 2008-2010 registra uno de -0.093\%; es decir, se mantuvo más estable en la

43. León-Manríquez, 2011.

44. Ídem. 


\section{Cuadro 2}

Estudio comparativo del volumen de las exportaciones

\begin{tabular}{lrrrrrrr}
\hline \multicolumn{3}{c}{ Canadá: Destino Exportaciones } & \multicolumn{3}{c}{ China: Destino Exportaciones } \\
\hline & 2008 & 2009 & 2010 & & 2008 & 2009 & 2010 \\
\hline Estados Unidos & 370.0 & 271.1 & 296.6 & Estados Unidos & 191.6 & 172.9 & 187.2 \\
Japón & 11.7 & 8.8 & 9.7 & Hong Kong & 147.7 & 95.3 & 127.7 \\
Reino Unido & 14.0 & 13.0 & 16.9 & Japón & 90.6 & 82.3 & 85.4 \\
Países europeos & 25.1 & 19.0 & 19.4 & República de Corea & 52.6 & 37.3 & 44.0 \\
Países de la OcDE & 20.7 & 16.6 & 17.9 & Alemania & 48.7 & 43.6 & 49.1 \\
Todos los demás & 47.0 & 40.6 & 44.00 & Países Bajos & 38.0 & 27.5 & 36.0 \\
países & & & & & & & \\
\hline
\end{tabular}

Fuente: Statistics Canada (http://www. Fuente: HKTDC (http://www.hktdc.com/ statcan.gc.ca/tables-tableaux/sum-som/101/ info/mi/ccs/en/China-Statistics.htm); cst01/gblec02a-eng.htm). General Administration of Customs of the People's Republic of China (http://www. customs.gov.cn/publish/portal0/tab44604/ module109000/info159797.htm).

crisis financiera asiática, pero alcanza un máximo frente al dólar en la crisis de 2008-2010.45

Ello se debió a la iniciativa de Beijing de mantener vinculado el yuan con el dólar estadounidense, evitando depreciar la moneda y que ello conllevara a otra oleada de devaluaciones competitivas monetarias en la región, ${ }^{46}$ pero dicha acción obedeció más a una lógica de protección del sistema económico doméstico, que a un intento de "contagiar" de estabilidad monetaria al resto de los países asiáticos.

Lo anterior es importante ya que refleja un obstáculo para que China no pueda construir regímenes monetarios por sí sola. Estados Unidos había argumentado que dicha vinculación, ligada a su vez a un fuerte control estatal, era culpable del gran déficit comercial que registraba el país americano respecto del asiático; no obstante, el déficit comercial obedece más a una lógica estructural interna de la economía estadounidense, aunada a los impactos de la globalización y a la carga política que impone Washington al déficit bilateral, ${ }^{47}$

45. Batson, Andrew, Hong, Shen, y Shaw, Joy (2010), "El yuan toca un nuevo máximo frente al dólar con nuevo sistema cambiario", Lanacion.com

46. YiFu Lin, 2011: 5.

47. Li, 2008. 
situación que Estados Unidos parece ha podido comprender, retirando las acusaciones que por más de una década le impuso al gobierno de Beijing, ${ }^{48}$ aunque sin dejar de considerar a la moneda como subvaluada o infravalorada. ${ }^{49}$

En materia de comercio, se observa en el segundo cuadro que, con excepción de Malasia, los países del Asia-5 registran importantes reducciones en su balanza comercial de bienes respecto del mundo. Pero todos ellos, con énfasis en Corea del Sur y exceptuando a Malasia otra vez, registran incremento de su comercio de bienes para 2009 y se mantiene estable en 2010 (con excepción de Tailandia). Lo anterior puede mostrar que en efecto se recupera el dinamismo comercial. El único caso que no presume de ello es Filipinas, pero eso se debe a otras cuestiones que no tienen nada que ver con la crisis, ya que ese país venía registrando un saldo negativo de bienes desde 2000, pero no se podría decir lo mismo de los servicios.

El caso de China es interesante puesto que de 2000 a 2004 registraba un comercio estable y a partir de ese año empieza a repuntar de manera incesante, llegando a un máximo histórico en 2008 con 297,040 millones de dólares en el intercambio de bienes. No obstante, es a partir de este año que se nota un descenso en las exportaciones chinas, aunque ello pudiera obedecer a otros factores: a) la tendencia al proteccionismo de parte de grandes mercados como Estados Unidos y Europa; b) la decisión de China de "frenar un poco" su crecimiento anual con base en las exportaciones, y c) fomentar el consumo interno. ${ }^{50}$

Con base en lo anterior, ¿se podría afirmar que en esta ocasión la República Popular ya puede fomentar regímenes y/o instituciones internacionales para la provisión de un BPG? Eminentemente prematuros, los hechos denotan que aún no posee esa habilidad hegemónica.

Ahondando un poco al respecto, aquí surge una problemática: citando a Kindleberger y Aliber: "los detalles proliferan, la estructura permanece"; 51 la dinámica capitalista global ha permitido realizar cambios sistémicos, pero no

48. "Us declines to cite China as currency manipulator", CBS, 2012, en http://www.cbsnews. com/8301-505245_162-57441630/us-declines-to-cite-china-as-currency-manipulator/

49. "Us says China's yuan undervalued, not manipulated", Channel News Asia, 2012, en http:// www.channelnewsasia.com/stories/afp_world_business/view/1203656/1/.html

50. Véanse por ejemplo: Qingfen y Ying, 2012; y "Progress, stability targeted as Parliament session starts", Xinhua, 2012, en http://news.xinhuanet.com/english/china/201203/05/c_131446519.htm

51. Kindleberger y Aliber, 2011: 34. 


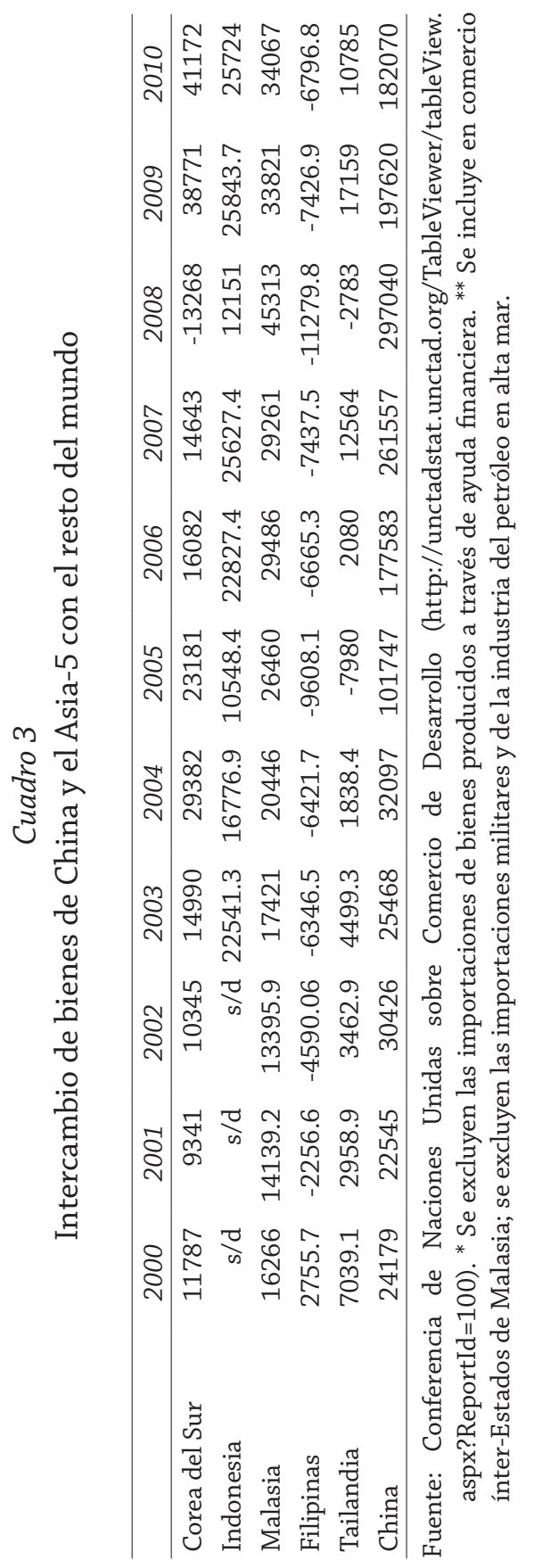


cambios de sistema, y cuando éstos suceden, la distorsión en la estructura global es tal que se produce inestabilidad, lo cual lleva a conflictos entre las potencias que quieren cambiar el sistema a su favor.

Esto se complementa por la puntual descripción que realiza Hoogvelt de las múltiples crisis capitalistas por las que ha pasado el sistema mundial desde que los mercados de valores emergieron por primera vez en Ámsterdam (Países Bajos) por allá del siglo XVII, pasando por la burbuja del Mar del Sur en la década de 1720 (Londres, Gran Bretaña), la crisis de la deuda latinoamericana un siglo después (esto vinculado a su vez con fenómenos como la fiebre del oro y las manías de los vías férreas y canales interoceánicos), el Jueves Negro y la Gran Depresión en 1929 y, obviamente, la Crisis Financiera Asiática de 1997 y la Gran Recesión Mundial de 2008. ${ }^{52}$

Como ya se había resaltado en la delimitación teórica de los BPG, el cambio del patrón oro al patrón dólar fue parte de una alteración más profunda que presenció el sistema mundial de 1919 a 1945, cuando la Pax Britannica había llevado su capacidad hegemónica al límite, dando paso a la Pax Americana, cuya hegemonía modificó las estructuras mundiales a su favor, ello reflejado en las instituciones de Bretton Woods y del Sistema de Naciones Unidas.

Coincido con Gilpin en cuanto a que se necesita un poderoso sustento político que sea capaz de respaldar una economía internacional próspera y estable, al igual que cualquier economía doméstica. Lo anterior se ve mermado a causa de las manifestaciones de la contradicción que implica una economía planetaria cada vez más integrada vis á vis, una enorme fragmentación política que cada vez más se acentúa con aparición de nuevos Estados nacionales. ${ }^{53}$

Es decir: la integración de la economía mundial avanza más rápido y con mayor dinamismo que la integración política, ${ }^{54}$ la cual pareciera ir al lado contrario, y cuya expresión posiblemente más clara es aquélla del fomento de regionalismos, pues los países presentan integraciones económicas, más que políticas.

52. Hoogvelt, 2010: 55 .

53. Gilpin, 2003b: 39 y 40.

54. Un ejemplo excelente me parece que es el de la Unión Europea, cuya inhabilidad para consolidar la integración política con base en la Constitución Europea no le ha permitido enfrentar con mayor éxito la crisis global financiera de 2008-2010, discutiendo incluso la posible desaparición del euro y la separación de Grecia, entre otras drásticas medidas. 
Ya hablando del caso específico de la República Popular China, como lo señalan Cornejo y Navarro, ${ }^{55}$ pareciera que las medidas que le ayudaron a paliar la crisis asiática, de nueva cuenta le sirven para la recesión global: fuerte posición fiscal y control sobre el sector financiero, aunque como ya se había señalado líneas arriba, también se fomentó el consumo doméstico aprovechando la vastedad del mercado interno.

Cornejo también estipula que el gobierno chino tuvo una excelente respuesta mostrada en el incremento del gasto público en fundamentalmente siete rubros: infraestructura urbana (bienes raíces de interés social), infraestructura rural, infraestructura para comunicaciones y transportes, salud y educación, protección al ambiente, innovación técnica, y reestructuración postsísmica en la provincia de Sichuan. ${ }^{56} \mathrm{Al}$ parecer se pudieron proveer de bienes públicos locales sin ningún problema.

En el caso de los BPG, específicamente el de gobernanza por medio de la estabilidad financiera, China no parece estar fomentando algún régimen, ni menos una institución; al contrario, parece querer librarse de los malos efectos sin alterar el statu quo, ya que se ha beneficiado enormemente del mismo, y dos claros ejemplos de ello son su comercio con base en las exportaciones, y el valor de su moneda al estar vinculada al dólar.

No ha propuesto, como el caso japonés en la crisis asiática, un fondo monetario asiático, ni un régimen monetario con base en el yuan, o instituciones financieras con base en un "modelo de Shangai", ni ha emprendido acciones

55. Cornejo y Navarro, 2011.

56. Ídem. 
agresivas aprovechando la debilidad financiera estadounidense para ascender un peldaño más hacia la hegemonía global.

En esta línea de pensamiento, cabe destacar que China alimenta la contradicción aparente entre el sistema económico y político global contemporáneo, ya que pugna por un nuevo orden con base en la multipolaridad (duojihua) y un "modelo estatista". ${ }^{57}$ Es decir, China parece apostar por regresar a un orden westfaliano en lo económico sin revolucionar las estructuras. Y la propuesta china se suma a aquélla de las "potencias emergentes", donde el grupo más conocido es el de los BRIC, ${ }^{58}$ y de cuyo seno han salido las críticas más contundentes para el rediseño (mas no revolución) de la arquitectura financiera global. Tan sólo recordar las declaraciones del presidente Hu Jintao en la cumbre del G-20 sobre la necesidad de resolver la crisis global de 2008-2010 desde una perspectiva multilateral, sumado ello a las audaces propuestas hechas desde los BRIC, de las cuales la más interesante es la de quitar al dólar como divisa mundial, y en su lugar colocar los derechos especiales de giro del FMI, incluidas ya las monedas de los BRIC. ${ }^{59}$ De hecho se había propuesto la creación de una banca BRIC, aunque tal iniciativa no contó con el impulso necesario, ya que el grupo supra mencionado aún no tiene una estructura que encauce su cooperación hacia una misma dirección. ${ }^{60}$

Como conclusión a este apartado, frente a la recesión global financiera de 2008-2010 Beijing no está promoviendo ningún nuevo régimen con base en una institución mundial que cambie la estructura a su favor. Con dificultades pudo enfrentar el impacto del interior (y eso por tratarse del sector financiero, a lo cual está muy protegida), pero no se hizo el efecto de spillover necesario para proveer del BPG gobernanza con base en la estabilidad financiera. Y ello no la hace una hegemonía en ascenso, al menos en el ámbito de la provisión de BPG.

57. Zhao, 2008: 27; Chin, Gregory y Thakur, 2010: 122.

58. Brasil, Rusia, China, India y Sudáfrica.

59. Véanse: "Análisis: China coopera para resolver la crisis financiera mundial", Spanish.china. org.cn; "China pide a FMI añadir monedas BRIC", PYMEX, 2011; "Exigen BRICS modernizar el sistema monetario", El Economista, 2011.

60. Véase: "Los BRICS estudian abrir un banco mundial alternativo", BBC Mundo, 2012; Devraj, 2012. 


\section{Consideraciones finales}

En esta investigación se encontraron resultados por demás interesantes que vale la pena compartir, y que tratan sobre la dinámica económico-política global. La hegemonía estadounidense no desempeñó su papel en la crisis asiática, pero eso no implicó un debilitamiento determinante en su poder estructural, por lo que siguió siendo la hegemonía global durante otra década (ello se refleja, por ejemplo, en la invasión a Irak sin la venia del Consejo de Seguridad de la ONU).

En 1997, y como se constató en este estudio, China se mantuvo un poco al margen y sus acciones fueron pequeñas o nulas respecto al mejoramiento de la situación. Japón tuvo todas las posibilidades de consolidar un liderazgo regional que poco a poco venía construyendo con el "modelo de vuelo de gansos", pero debido a restricciones domésticas (como los obstáculos que implican las pugnas entre distintos grupos de poder) y externas (la dependencia militar y política respecto a Estados Unidos), sus iniciativas no tuvieron el efecto deseado y su presencia se vio disminuida en favor de China. ${ }^{61}$

Frente a los actores individuales emergían intentos de un nuevo multilateralismo cuya base regional (y no universal como el sistema ONU) se tradujo en una mejor respuesta para combatir los efectos de la crisis, y aunque no lograron disminuirla, sí la contuvieron y sus efectos no se hicieron mundiales. Luego entonces se observa una estructura económica que, dada su complejidad, sobrepasaba las capacidades estatales y los únicos entes capaces de controlarla fueron aquéllos multilaterales.

Lo dicho en el párrafo anterior se ve de manera más clara en el caso de la recesión de 2008. De inicio, habría que aclarar que la naturaleza de ambas crisis capitalistas son diferentes: la primera fue fundamentalmente monetaria y afectó la estabilidad de las balanzas de pagos de los países del sureste asiático; la segunda fue bancaria y pronto contagió al sistema financiero internacional, y a su vez afectó al comercio mundial. Aunado a esto, la primera se originó en un país en desarrollo y la segunda en un país desarrollado, y no sólo eso, sino en el país hegemónico desarrollado, la Pax Americana.

Así, la respuesta multilateral se visualiza de manera más clara y contundente. En este caso no hay intentos por parte de Estados individuales (como

61. Ramírez Bonilla, 2011: 103-150. 


\section{Figura 2}

Dinámica de los actores, crisis financiera asiática 1997-1999

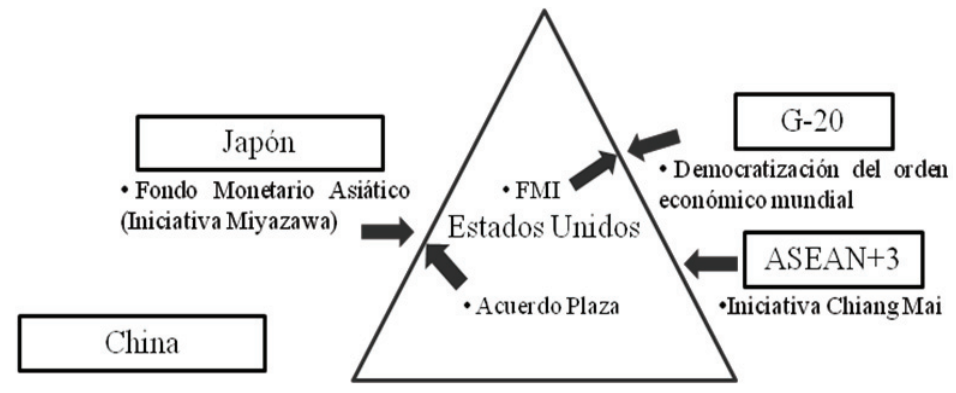

Hegemonía mundial

Fuente: elaboración propia con base en la presente investigación.

fue el caso de Japón) de subsanar al sistema tratando proveer algún BPG; en su lugar, los BRIC y el G-20 parecen ser los únicos mecanismos adecuados para proveer dichos bienes. Y aunque China, por ejemplo, haya ofrecido a la Unión Europea comprar parte de su deuda, se debe recordar que Beijing ofreció dicha ayuda en el marco de un FMI mejor reestructurado, además de que de manera clara sólo se ofreció a asistir en la compra de las deudas de algunos países miembros, no de toda la Unión.

\section{Figura 3}

Dinámica de los actores, recesión global financiera 2008-2010

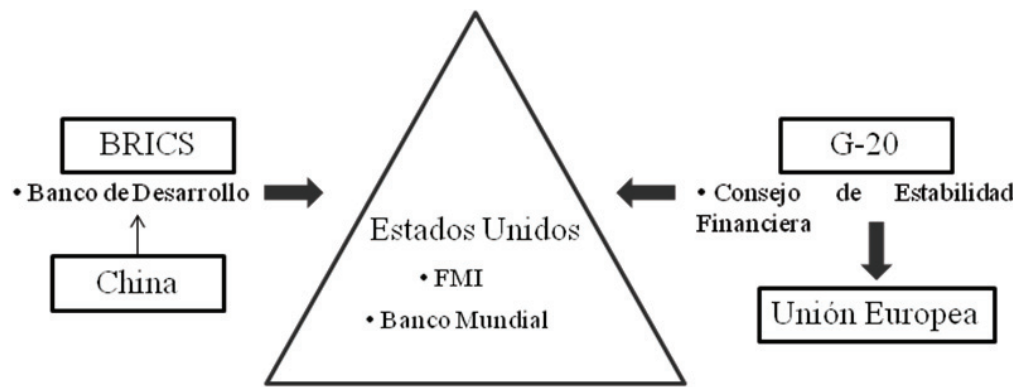

Hegemonía mundial

Fuente: elaboración propia con base en la presente investigación.

¿Qué infiero de todo esto? Beijing no orienta su política exterior hacia la consolidación de una hegemonía global, en razón de que no tiene los recursos 
para hacerlo. Además, lo anterior se debe también al carácter cauteloso que la dirigencia china ha tenido desde la apertura económica, lo que en palabras de Deng Xiaoping sería: "hay que cruzar el río pisando las piedras". Pero también se debe a que el dinamismo económico-comercial aumenta tan rápido, que se sale del control de los Estados, presenciando fuertes distorsiones a causa de que el comercio mundial se incrementa más rápidamente que la producción mundial, dando origen a un "subconsumo" y a una provisión excesiva de bienes manufacturados. ${ }^{62}$

Aun cuando Estados Unidos cuenta con un enorme poder estructural, el sistema mundial ha crecido de una manera tan desproporcionada respecto al poder de la hegemonía para mantenerlo, que la crisis de 2008-2010 es el primer signo (y probablemente no será el último) de que las capacidades nacionales estadounidenses proyectadas al ámbito global ya no son suficientes o adecuadas para un sistema que el mismo Estados Unidos creó, y cuya base es un exacerbado consumismo. Como ya preveía Gilpin: "la fe estadounidense prevaleciente en la mano invisible del mercado puede que sea socavada por inesperados eventos mientras avance el siglo XXI" ${ }^{63} \mathrm{Y}$ ese evento fue precisamente la crisis global financiera.

En esta investigación me propuse comparar el papel de China en las crisis financieras de 1997 y de 2008 para comprobar si su desempeño implicaba el rasgo hegemónico de la provisión del bien público global de la gobernanza, sumado al bien complementario de la estabilidad financiera. Para ello me remito a la hipótesis presentada en mi resumen: resulta ser cierto que en la crisis de 1997-1999 China no presentó rasgos hegemónicos con base en la provisión de algún BPG, pero resultó ser falso que en la crisis 2008-2010 sílos presenta, toda vez que las propuestas sobre regímenes internacionales para enfrentar los efectos de la crisis, se han propuesto desde bases multilaterales, además de que las secuelas de dicha crisis, si no se resienten en el sistema económico-comercial, sí en la arquitectura financiera mundial.

Esta investigación se suma a otra efectuada previamente para indagar sobre la posibilidad de que Beijing estuviera en un tránsito de "hegemonía en ascenso", donde en un análisis multifactorial con base en capacidades militares, tecnológicas, económicas, de poder suave, y domésticas, también se

62. Gilpin, 2003b: 57.

63. Gilpin, 2003a: 80. 
demostró que el país asiático no presenta rasgos hegemónicos de "potencia en ascenso". ${ }^{64}$ miv

\section{Referencias bibliográficas}

Abeles, Martin, y Kiper, Esteban (2010), El G20: ¿Hacia una nueva arquitectura financiera internacional? El rol de Argentina-México-Brasil, ponencia presentada en el marco del Observatorio de Política Exterior Argentina, Asociación de Economía para el Desarrollo de Argentina y CEPEs, Buenos Aires. Acjkgöz Ersoy, Bernur (2011), "Globalization and Global Public Goods", en Pachura, Piotr (Ed.), New Knowledge in a New Era of Globalization, Tech. Recuperado de: <a href="http://www.intechopen.com/books/newknowledge-in-a-new-era-of-globalization/globalization-and-global-public-goods" title="globalization-and-global-public-goods" $>$ Globalization and Global Public Goods $</ a>$

Ayala Espino, José (2000), Instituciones y economía. Una introducción al neoinstitucionalismo económico, México: FCE.

Bustelo, Pablo (1999), "Las peculiaridades de Asia Oriental: Desarrollo económico, crisis financieras y perspectivas en el siglo XxI”, Mercurio: Revista de Economía y Empresa, núm. 4.

- - (2000), "La economía china en las crisis asiáticas", Economía Exterior, núm. 13, España: Universidad Complutense de Madrid.

- - (2001), "La economía china durante y después de las crisis asiáticas (1997-2000)", en Cesarín, Carlos, y Moneta, Carlos (comps.), China: Perspectivas del presente, desafíos del futuro, Buenos Aires: Eduntref.

Cesarín, Carlos, y Moneta, Carlos (comps.) (2001), China: Perspectivas del presente, desafíos del futuro, Buenos Aires: Eduntref.

Cornejo, Romer, y Navarro, Abraham (2011), "China y la crisis mundial. Efectos y reacciones”, La crisis global de 2008-2010. Respuesta en las Américas y el Este asiático, Seminario Internacional, México: Universidad Autónoma Metropolitana-Xochimilco.

Chin, Gregory, y Thakur, Gregory (2010), "Will China Change the Rules of the Global Order?", The Washington Quaterly, 33(4).

Eichengreen, Barry (2004), "Hanging together? On monetary and financial cooperation”, en Yusuf, Shahid, Altaf, Anjum M., y Nabeshima, Kaoru

64. Véase: Tzili Apango, 2012. 
(eds.), Global change and East Asia Policy Initiatives, Estados Unidos: World Bank/Oxford University Press.

Fawcett, Louise, y Hurrell, Andrew (1995), Regionalism in World Politics, Estados Unidos: Oxford University Press.

Fernald, John G., y Babson, Oliver D. (1999), "Why has China survived the Asian crisis so well? What risks remain?”, International Finance Discussion Papers, Estados Unidos: Board of Governors of the Federal Reserve System. Ferroni Marco, y Mody, Ashoka (eds.) (2002), International Public Goods. Incentives, Measurement and Financing, Estados Unidos: The World Bank/ Kluwer Academic Publishers.

García Arias, Jorge (2004), "Un nuevo marco de análisis para los bienes públicos: La teoría de los bienes públicos globales", Estudios de Economía Aplicada, 22(2), España.

Gilpin, Robert (1988a), "The Theory of Hegemonic War", Journal of Interdisciplinary History, 18(4), Estados Unidos: The Origin and Prevention of Major Wars/The MIT Press.

- - (1988b), War and Change in World Politics, Reino Unido: Cambridge University Press.

- - (1990), La economía política de las relaciones internacionales, Argentina: Grupo Editorial Latinoamericano.

- - (2003a), "A Postscript to the Asian Financial Crisis: The Fragile International Economic Order", Cambridge Review of International Affairs, 16(1).

- - (2003b), El reto del capitalismo global: La economía mundial en el siglo XXI, España: Océano.

Haggard, Stephen, y Simmons, Beth A. (1987), "Theories of International Regimes”, International Organization, 41(3).

Hoogvelt, Ankie (2010), "Globalisation, Crisis and the Political Economy of the International Monetary (Dis)Order", Globalizations, 7(1-2), Estados Unidos: Routledge.

Hsiung, James C. (2009), “China's Second Ascent and International Relations Theory", APSA.

Hurrell, Andrew (1992), “Teoría de regímenes internacionales. Una perspectiva europea”, Foro Internacional, núm. 130, xxxiI(5), México: El Colegio de México.

Kaul, Inge, Grunberg, Isabelle, y Stern, Marc A. (eds.) (1999), Global Public Goods. International Cooperation in the $21^{\text {st }}$ Century, Estados Unidos: The United Nations Development Programme. 
Keohane, Robert (2005), “Interdependencia, cooperación y globalismo”, en Borja Tamayo, Arturo (comp.), Ensayos escogidos, Col. de Estudios Internacionales, México: CIDE.

Kim, Chi-Wook (2011), "El G20 y los cambios en la gobernanza global”, Revista Mexicana de Política Exterior, núm. 91, México: Instituto Matías Romero. Kindleberger, Charles (1986), "International Public Goods without International Government", The American Economic Review, 76(1).

Kindleberger, Charles P., y Aliber, Robert Z. (2011 [1978]), Maniacs, Panics and Crashes. A History of Financial Crisis, $6^{\text {a }}$ edición, Estados Unidos: Palgrave Macmillan.

Kwan, C. H. (1998), "The Yen, the Yuan, and the Asian currency crisis. Changing fortune between Japan and China", A/PARC Ocassional Paper, Stanford: Shoresteing APARC.

León-Manríquez, José Luis (coord.) (2011), La crisis global de 2008-2010. Respuesta en las Américas y el Este asiático, Seminario Internacional, México: Universidad Autónoma Metropolitana-Xochimilco.

$\mathrm{Li}$, Wei (2008), "China-U. S. economic relations and the trade imbalance issue", en Zhao, Suisheng (ed..), China-US Relations Transformed. Perspectives and strategic interactions, Estados Unidos: Routledge Taylor \& Francis Group. Estados Unidos.

López Villafañe, Víctor (1999), Asia en transición: Auge, crisis y desafíos, México: Siglo XXI Editores.

Nossel, Suzanne (2004), “Smart Power”, Foreign Affairs, 82(2), Estados Unidos. Nye, Joseph S. Jr. (2011), The Future of Power, Estados Unidos: Public Affairs. Oliver, Morrisey, Willem te Velde, Dirk, y Hewitt, Adrian (2002), "Defining International Public Goods: Conceptual Issues”, en Ferroni, Marco, y Mody, Ashoka (eds.), International Public Goods. Incentives, Measurement and Financing, Estados Unidos: The World Bank/Kluwer Academic Publishers. Polanyi, Karl (2003 [1944]), La gran transformación, México: FCE.

Qinglian, He (1997), "China faces financial crisis at the turn of the century", U. S.-China Economic and Security Review Comission. Recuperado de: http:// www.uscc.gov/researchpapers/2000_2003/pdfs/chinfac.pdf

Ramírez Bonilla, Juan José (2011), "Japón y la Asociación del Sureste de Asia. Una relación ambiciosa en el ámbito retórico, limitada en el práctico”, en Ramírez Bonilla, Juan José, Toledo Beltrán, Daniel, y Uscanga Prieto, Carlos (coords.), Japón ante la configuración de Asia del Pacífico. Proactividad y reactividad ante un orden internacional fluido, México: El Colegio de México-Centro de Estudios de Asia y África, pp. 103-150. 
Ramírez Bonilla, Juan José, Toledo Beltrán, Daniel, y Uscanga Prieto, Carlos (coords.) (2011), Japón ante la configuración de Asia del Pacífico. Proactividad y reactividad ante un orden internacional fluido, México: El Colegio de México-Centro de Estudios de Asia y África.

Sandler, Todd (1998), "Global and Regional Public Goods: A Prognosis for Collective Action”, Fiscal Studies, 19(3), Estados Unidos.

Singh, Ajit (1998), "Asian Capitalism" and the Financial Crisis", CEPA Working Paper Series III, Working Paper, núm. 10, Estados Unidos: Center for Economic Policy Analysis.

Sitiglitz, Joseph (2003), Globalization and its discontents, Estados Unidos: W. W. Norton \& Company.

Tzili Apango, Eduardo (2012), ¿Pax Sinica? Viabilidad de la aplicación del modelo de Robert Gilpin para analizar el "ascenso de China", tesis de licenciatura, México: BUAP-Facultad de Derecho y Ciencias Sociales.

YiFu Lin, Justin (2011), "China and the Global Economy", Asia's Role in the Post-Crisis Global Economy, conferencia impartida en la Reserva Federal, Estados Unidos.

Yusuf, Shahid, Altaf, Anjum M., y Nabeshima, Kaoru (eds.) (2004), Global change and East Asia Policy Initiatives, Estados Unidos: World Banks/ Oxford University Press.

Zhao, Suisheng (ed.) (2008), "China rising. Geo-strategic thrust and diplomatic engagement", China-Us Relations Transformed. Perspectives and strategic interactions, Estados Unidos: Routledge Taylor/Francis Group.

Fuentes electrónicas

"A Stable Yuan Contributes to the Global Economic Recovery", China. org.cn, 2010, en http://www.china.org.cn/report/2010-03/01/content_19497854.htm

"Análisis: China coopera para resolver la crisis financiera mundial", Spanish. china.org.cn, en http://spanish.china.org.cn/specials/091dfh/2009-04/03/ content_17551013.htm

“China pide a FMI añadir monedas BRIC", PYMEX, 2011, en http://www.pymex. pe/Mundo/china-pide-a-fmi-anadir-a-monedas-bric.html

"Exigen BRICS modernizar el sistema monetario", El Economista, 2011, en http://eleconomista.com.mx/economia-global/2011/04/14/exigen-bricsmodernizar-sistema-monetario 
"Los BRICS estudian abrir un banco mundial alternativo", BBC Mundo, 2012, en http://www.bbc.co.uk/mundo/noticias/2012/03/120328_nuevo_banco_brics_ar.shtml

"Pro-Active Policies by China in Response to Asian Financial Crisis", Ministry of Foreign Affairs of the People's Republic of China, 2000, en http://www. fmprc.gov.cn/eng/ziliao/3602/3604/t18037.htm

"Progress, stability targeted as Parliament session starts", Xinhua, 2012, en http://news.xinhuanet.com/english/china/2012-03/05/c_131446519. htm

"Protectionism won't help", China Daily, 2012, en http://www.chinadaily.com. cn/cndy/2012-02/01/content_14514623.htm

"Us declines to cite China as currency manipulator", CBS, 2012, en http:// www.cbsnews.com/8301-505245_162-57441630/us-declines-to-citechina-as-currency-manipulator/

"Us says China's yuan undervalued, not manipulated", Channel News Asia, 2012, en http://www.channelnewsasia.com/stories/afp_world_business/ view/1203656/1/.html

Conferencia de Naciones Unidas sobre Comercio de Desarrollo, 2011, en http:// unctadstat.unctad.org/TableViewer/tableView.aspx?ReportId=100

Devraj, Ranjit (2012), "BRICS posterga creación de su banco", IPS, en http:// ipsnoticias.net/nota.asp?idnews $=100451$

Financial Stability Board, en http://www.financialstabilityboard.org/about/ history.htm

Fondo Monetario Internacional, 2012, en http://www.imf.org/external/np/ fin/data/param_rms_mth.aspx

General Administration of Customs of the People's Republic of China, 2012, en http://www.customs.gov.cn/publish/portal0/tab44604/module109000/ info159797.htm

HKTDC, 2012, en http://www.hktdc.com/info/mi/ccs/en/China-Statistics.htm Jianxin, Lu (2008), "Risks limit depreciaton of yuan", The New York Times, en http://www.nytimes.com/2008/12/03/business/worldbusiness/03ihtcol04.1.18360084.html

Qingfen, Ding, y Ying, Diao (2012), "Domestic consumption takes the driver's seat", People's Daily Online, en http://english.peopledaily.com. cn/90778/7765636.html

Statistics Canada, 2012, en http://www.statcan.gc.ca/tables-tableaux/sumsom/101/cst01/gblec02a-eng.htm 


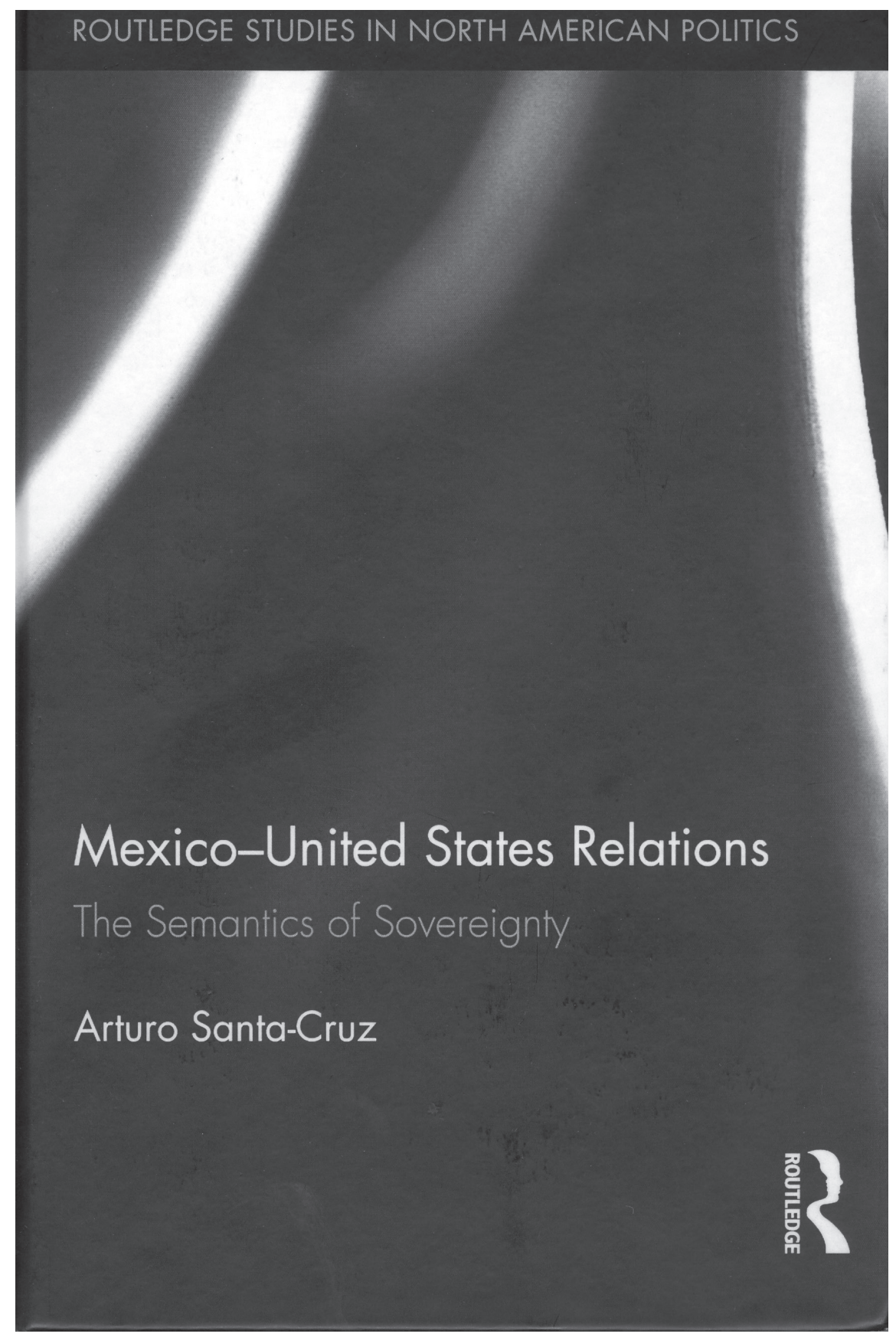

